\title{
Standardization on Framework and Scenarios for Self-directed Ubiquitous Learning (Y.fsul)
}

\author{
Young Jun Kim*, Sokpal Cho** and Ilyoung Chong * \\ *Dept. of Information and Communications Engineering, Hankuk University of Foreign Studies, Seoul, Korea \\ **Dept of Information Communications, Sungkyul University, An Yang, Korea \\ ddanggae@hufs.ac.kr, spcho@sungkyul.edu, iychong@hufs.ac.kr
}

\begin{abstract}
- Abstract - The paper addresses a standardization progress on service framework and scenario for establishing self-directed u-learning (Y.fsul) applications in accordance with ubiquitous ICT (Information and Communications Technology) environments. The Draft Recommendation Y.fsul covers the service framework for self-directed u-learning in ubiquitous ICT environment (e.g., smart virtual space, smart WoO provisioned space, and etc.) and service model for self-directed u-learning service scenarios
\end{abstract}

Keywords - u-learning, object, self-directed, semantic ontology, collaboration

\section{INTRODUCTION}

A u-learning environment is any setting in which learners can become totally immersed in the learning process. So, a ulearning environment is a situation or setting of pervasive or omnipresent education or learning. The self-directed learning environments can be accessed in various contexts and situations in terms of leaner's environments. Thus, it is necessary to be operable among entities in ubiquitous ICT environments.

This draft Recommendation (Y.fsul) identifies the following objectives for self-directed u-learning services in ubiquitous ICT environment:

- service framework for self-directed u-learning in ubiquitous ICT environment

- service model for self-directed u-learning service scenarios for self-directed u-learning

\section{A. How to describe what a u-learning entity is and does?}

The functionality of most u-learning entities (i.e. how to interact with it) for u-learning can be described using the following model:

- operations (methods, parameters, return u-learning entity), which can

- be synchronous or asynchronous (block call until a response is sent or not)

- allow concurrent accesses from other users or not

- event sources

- properties (management parameters), which can be:

- read-only

- read and write
The u-learning entity metadata description (which entity, where it is located.) can be described using:

- an unique u-learning entity identifier. It can be difficult with entities with no unique physical address and no persistence.

- an entity description using ontologies, e.g.:

- features: measured parameters

- context: location, ownership

Most parts of these descriptions are relative to the $\mathrm{u}$ learning entity class, some are specific to the u-learning entity instance.

B. What kind of interactions?

- event-based interactions:

- pull from a u-learning entity (e.g. request a entity from u-learning entity pool or DB)

- push to a u-learning entity (e.g. send an entity to ulearning user)

- subscribe to notifications from u-learning entity (e.g. receive a warning when the user has been prohibited to access.)

- store-and-forward: in case of temporary disconnection, messages can be stored and sent upon reconnection

- stream-based interactions (audio, video) will be considered

C. How will user-created applications communicate with ulearning entity?

Each u-learning entity will need a clear interface (or API) exposing its functionalities. U-learning users will not need to know the API to use the entity in their applications. Some high-level representation will mask its complexity. Note that a lot of the existing u-learning devices already have some support for configuration and provisioning, but typically at a level that is too complex for our target user base.

- candidate protocols: UPnP, DPWS, Web Services, REST, XMPP IO-Data, MMH/ETSI protocol (eg: UMONS-Kysoh MultiModeHub), JointSPACE (for Philips televisions),

- candidate data formats: SensorML, EEML.

- candidate semantic technology to capture and externalise the meaning of entity data: RDFa, GRDDL, OWL(-S)

D. How to share u-learning entities?

Just like media content, u-learning entity can be shared and have different access rights:

- private (available only to me) 
- available to a group

- publicly available

Some u-learning entities are difficult to use by several users in a simultaneous way (e.g. photo frame, robot, television, PTZ camera). It will be necessary to allocate policies (timeslots...)

\section{OVERVIEW OF SELF-DIRECTED UBIQUITOUS LEARNING}

Based on the information of various content attributes the semantic ontology based content objectification could be created. When objectifying the content, different users in $\mathrm{u}-$ learning are able to provide and to be provided with new services by combining or easily using of the objects. In figure V.1 shows the content of object structure based on the Semantic Ontology.

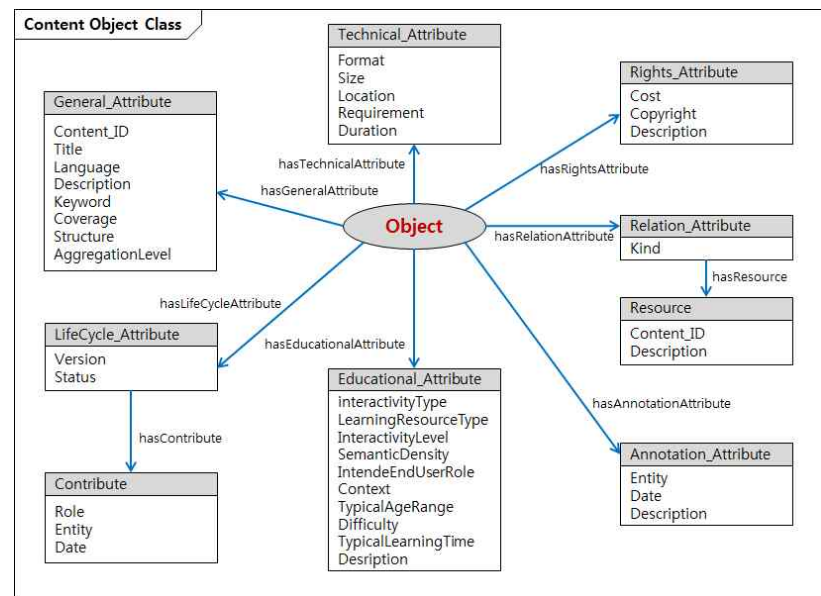

Figure 1. Semantic Ontology based Content Object Structure

Through the structure showed in the figure 1 the generated content objects are again combined with the other objects to create a new objects, and so various objects combines each other to provide and form a new service of u-learning.

And the figure 2 shows the content objectification, objectification that combines to create new objects and service construction of various object combinations by the demand of the users.

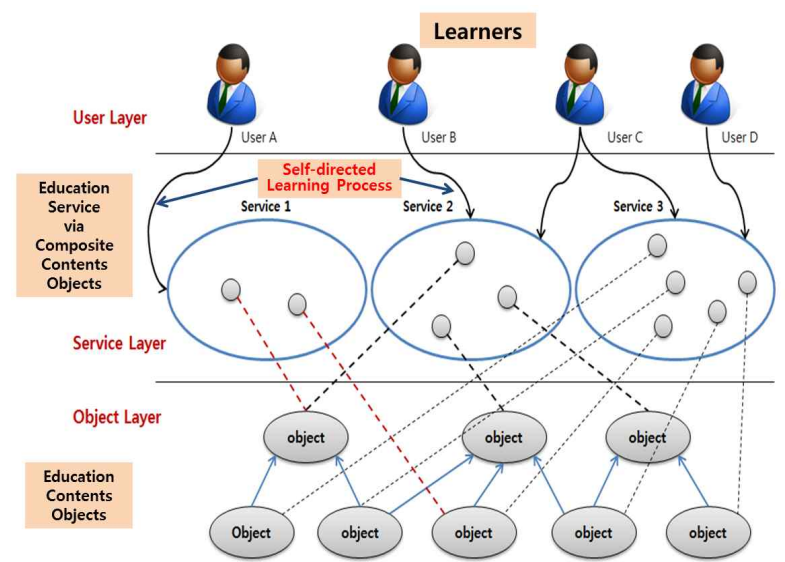

Figure 2. Objects Collaboration and Architecture of Ulearning Service
As of figure V.2, in order for the users to use the service are also able to construct a service, and these services are provided through the combination of different objects. And by then, other objects could be a previously constructed of collaborated objects. Such collaboration of objects is needed by all means to provide a user-centric u-learning service, due to the users' different environments and structures.

\section{III.OBJECTED SERVICE MODEL OF U-LEARNING}

The service model to provide u-learning in ICT environment has the general information of providing the users(learner/students) be able to review online based review learning section with resources(objects) which are openly offered on the web, and according to the users' selection it will be utilized to provide integrated review learning service. The various existing objects on the web environment are the data source for the learning purpose and each of the objects are included with specified metadata. The objects that are used to provide for the u-learning include text, video, media and different fields of omnipresent is provided to user according to their specific selection with prepared learning materials.

The figure 3. illustrates about the service model that each of the existing service that offer texts, images, videos, sounds and similar learning instruments of data are provided in content object form, and the user could meet the provision of comfortable learning environment by making its own settings in any circumstances due to present situation. Through ulearning service the user are able to realize a creative usercentric education and able to encounter custom and intellectual learning implementation according to the user's individual learning pretention.

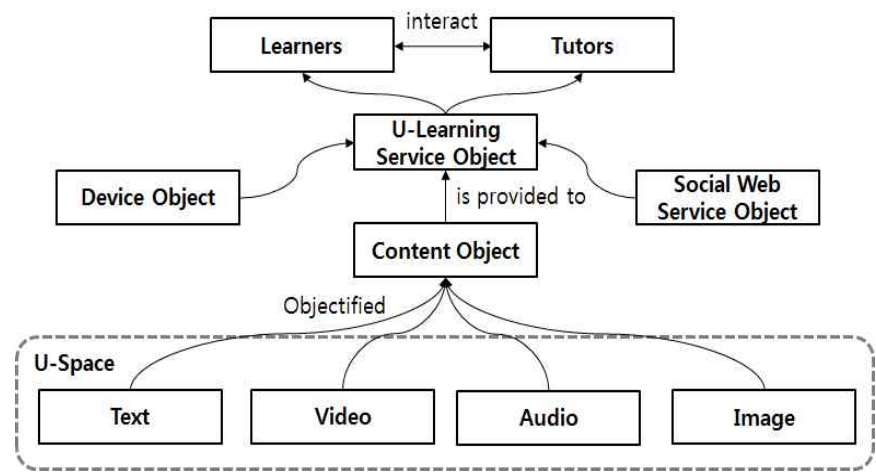

Figure 3. Objected Service Model for u-learning

The followings are the characteristics of U-learning service features:

- The educational cost could be reduced for having a universality features that is provided through the web with openly forms of content objects.

- The various content object could be simultaneously access capable, and even changes occurs due to the time alteration it will adjust and provide the service.

- The same content objects are provided to the user, but custom u-learning service could be held according to the requirements or characteristic of the users. 
- The information of content object has the scalability and having large ripple effect that could provide utilization and usefulness from the updated available information.

- The Just-in-Time learning service could be provided in any place according to the user's environmental location and the device terminal.

- Integrated u-learning service is capable to have interaction between the content objects that are collected from the user and the lecturer allowing them to collaborate by shares of knowledge and information with cooperation and increase good performance of learners.

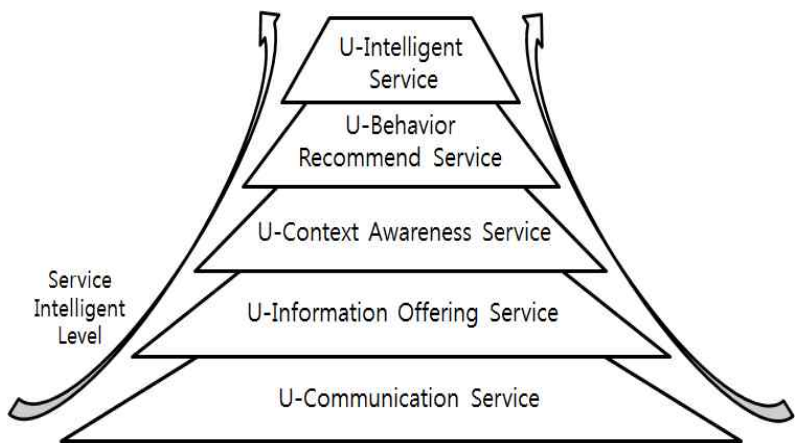

Figure 4. Education Service Level in Ubiquitous Environments

In order to produce the optimized and customized service of u-learning service in ubiquitous environment, the semantic elements are needed to provide the intelligent service by collecting and analysing the circumstance of a user's various environments.

The figure. 4 shows the various educational services according to the intelligent level of service in ubiquitous environment. In order for the u-learning service to produce a customized user-centric and intelligent service the high level of service functions are required to provide.

Accordance of ubiquitous service development the 5 kinds of educational services are listed in the following:

- u-communication service: ubiquitous network are utilized for neglecting the area constraints in order of enabling user's communication in any circumstances.

- u-information offering service: according to the user's demand real-time context are searched to provide service.

- u-context awareness service: a pre-defined situation is recognized and accordance to the user's demand the service information are provide in real-time.

- u-behavior recommend service: a service proposing the needed action from the user's inference demand

- $\mathrm{u}$-intelligent service: a service to provide convenience according to the user's location environment, according to the context the various information are identified intellectually, and automation.

\section{IV.Distributed ConetnTs ObJect MOdeL OF U- LEARNING}

The contents model in u-learning is depicted through the features of contents objects from an educational service perspective. As shown in figure 5, the object element should be published in the ontology repository to be consulted by learners. The content model of u-learning is composed of ContentObjectProfile,AttributeContainer,

ContentObjectMetadata, TimeFeature and Location to content the content model structure.

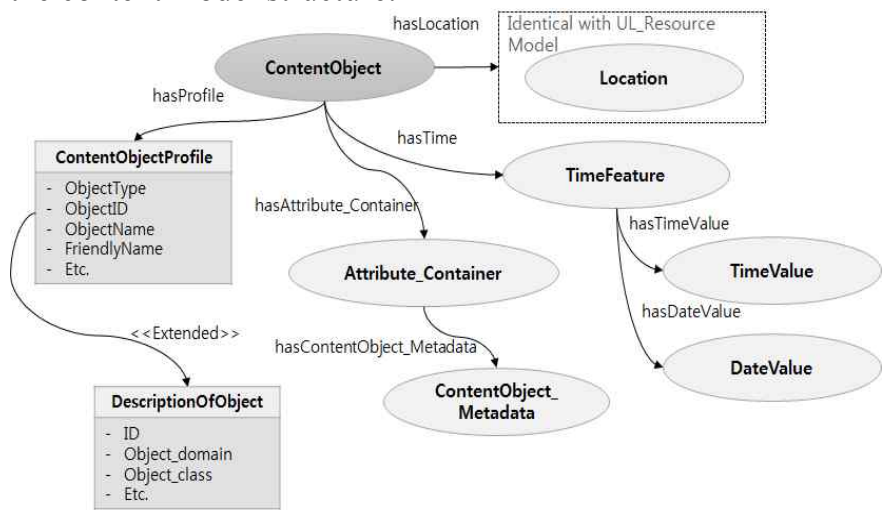

Figure 5. Content object model inside the ontology for selfdirected u-learning

- ContentObjectProfile consists of information that could support the profile of Content

- Location has the same identical model that is used in the UL_Resource model.

- TimeFeature indicates the time and the date specified as TimeValue and DateValue.

- AttributeContainer contains one or more contents of the object.

- ContentObjectMetadata specifies the detailed information that is used to define the data information stored in Attribute Container.

- DescriptionOfObject is an extend profile information that are needed and support self-directed u-learning.

\section{B. RESOURCE MODEL}

The Resource model for self-directed u-learning represents the details and related data through digital world. This model could provide the core components of related data to provided efficient and effective leading of decision to support the content object model and service model.

- Location has the same identical model that is used in the content object model.

- UL_ResourceType specifies the service that the image, text, audio and video that could provide information, and link to the resource type.

- UL_AccessInterface gives an access to the interface that specifies what technology interface to be use in the InterfaceType.

- UL_InterfaceType further specifies the set of instance that reflects the interface technology which are provided on the web.

- Description of UL_Resource indicates the information that could support the Resource model.

\section{A. Contents Model}




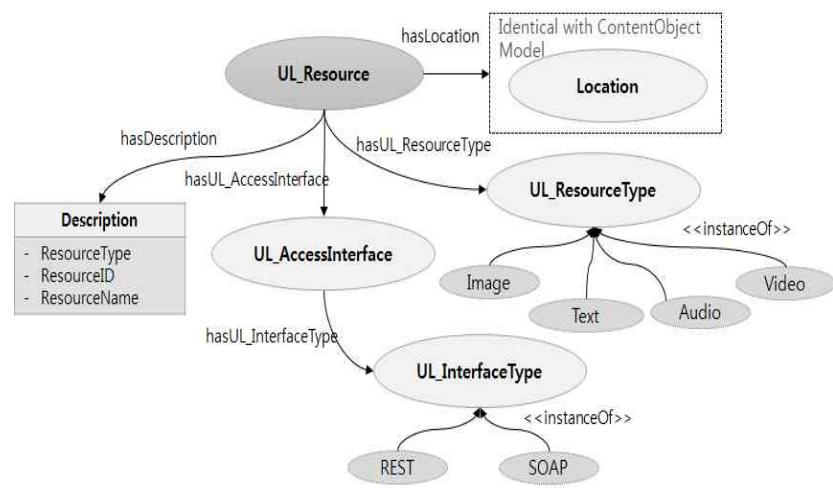

Figure 5. Example of Resource model inside the ontology for self-directed u-learning

\section{Semantic Profile of a Service in SELF-directed U- LEARNING SERVICE ENVIRONMENT}

To describe the semantic profile of services model for $\mathrm{u}$ learning, the objects in the u-learning service environment are described in the ontology model. The service may have several descriptive properties such as Input and Output PreCondition and effect (Post condition), and additional to that also includes non-functional properties such as Quality of Service, SLA and etc

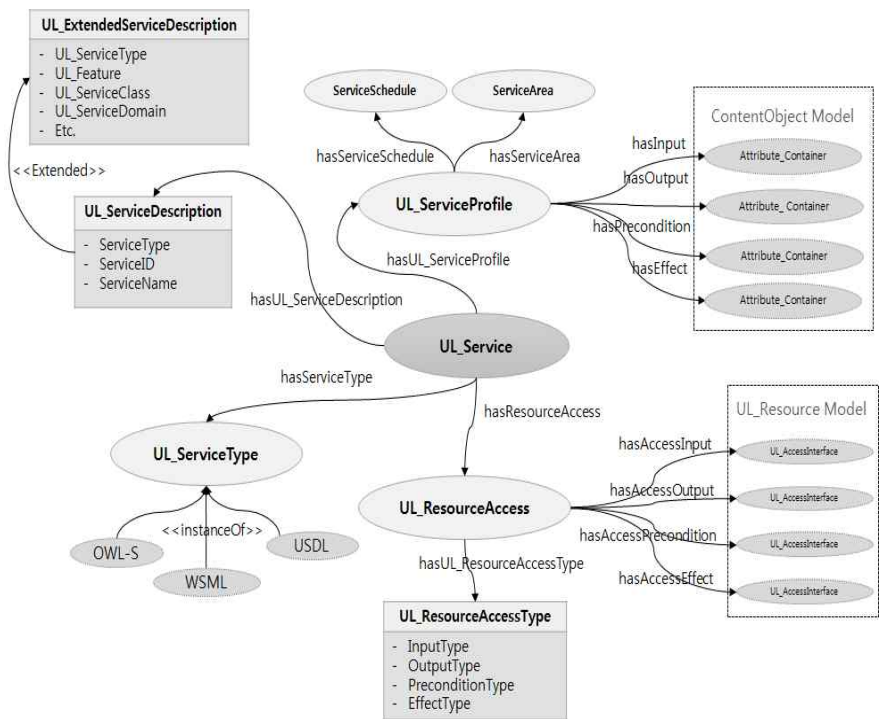

Figure 6. Service object model inside the ontology in ulearning environment

Service provides a well-defined and standardised interface, offering all necessary functionalities for interacting with contents object and related processes. The services expose the functionality of an UL content by accessing its hosted resources.

Illustrated model is a service semantic ontology model to provide service in u-learning environment. Indicating one service has a meaning of providing interoperable content in the service model, and the service model itself defines each of the available contents for interconnection. Also, by including the information of mapped resource model with contents model the needed access interface of a content object are provided during the service provision.

In order to provide semantic ontology based UL_services, the appropriate resource information can be accessed. As the resource service is mapped with content objects, based on resource information the appropriate contents are capable to controlled and managed.

In addition, to manage and to provide the resource service model in u-learning environment is responsible in linking the two models. The Service model is composed with four top properties as shown in the following description:

- UL_ServiceDescription: include the definition of the ulearning service, and u-learning service is also included as extension

- UL_ServiceType: the service explanation of modeling language type technique

- UL_ServiceProfile: the profile of service, range of service, schedule, input and output, precondition, effect and other related information are included. Also, the connection of each contents of u-learning to the ValueContainer in content object model

- UL ResourceAccess: having the information to access the information type and resource in approaching the resource model, and it is connected AccessInterface

\section{AN EXAMPLE OF U-LEARNING SERVICE FROM USER- LEVEL CREATION PERSPECTIVE: CONTENTTALE}

The ContentTale platform contains three main layers: the device layer, the execution layer and the creation or user definition layer. These three layers will be shortly described below. A more thorough description will be elaborated in the next section.

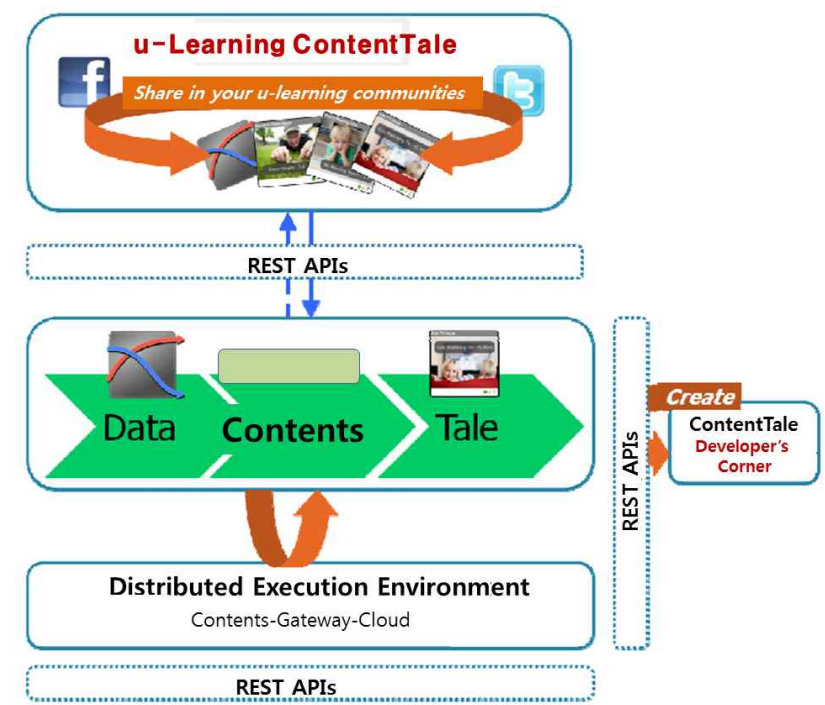

Figure 7. An example of user-level creation perspective in u-learnig: ContentTale

- U-learning entity layer 
- u-learning entity (e.g., contents) can be connected with the execution platform directly through a REST interface, through a u-learning gateway.

- u-learning entities communicate with the distributed execution platform through a REST interface posting JSON messages.

- Distributed execution environment

- The distributed execution environment is the place where the applications are actually deployed.

- Distributed - cloud

- applications

- set of specialized components for storage, status timers, exposure of the u-learning entities, u-learning entity transformation components

- set of rest interfaces for monitoring, deploying end-user defined applications, exposure of data through REST interfaces.

\section{- Third Party REST interfaces}

U-learning ContentTale provides a set of REST API's that allows third parties to connect to the platform from their own applications (see Figure VI.1). All data used in the APIs is described in JSON format. The APIs can be split in 3 parts:

1) push API's allows pushing data and status information to the ContentTale execution platform and retrieving raw or processed data out of it. Before you can start sending data you have to register your u-learning entity using the creation API which will give you the exact URL that you should use to send your data to.

2) creation API: provides methods to manage your sources/devices and define new source types. Below a non-exhaustive list of methods that can be called:

- Query for personal sources or sources shared by friends

- Deploy new sources/devices of a specified type

- Semantic search on the existing source types

- Creation of new data types (used to describe the data that is provided by a source)

- Creation of new source types

3) Visualization API: allow to request and embed full ContentTale visualizations in your own site.

\section{CONCLUSION}

The functional requirement for u-learning should include the following functions to form the simple mobile learning with the mobility and pervasiveness or omnipresent education, and to use the context awareness to provide most adaptive contents. In the technical aspect $u$-learning has to provide user assessable interface which offers adaptability to learner individual needs and learning styles.

And it has to fully support tracking of learning activity with mobility, and auto interface by language and visual aids. With more important, it should provide the learning process in the form of interactions with teachers and learners, and context management functions to relate different types of information when moving during the length of a trip or in route to school or home.
In order to provide its variety of u-learning service, the common framework to support its dynamic connectivity and delivery of digital educational contents will be sigificantly necessary.

\section{ACKNOWLEDGMENT}

This research was funded by MSIP(Ministry of Science, ICT \& Future Planning), Korea in the ICT R\&D Program 2013

\section{REFERENCES}

[1] [ITU-T Y.2060] Recommendation ITU-T Y.2060 (2012), Overview of the Internet of things, 2012

[2] [ITU-T Y.2063] Recommendation ITU-T Y.2063 (2012), Framework of Web of Things, 2012

[3] [ITU-T Y.3021] Recommendation ITU-T Y.3021 (2012), Framework of energy saving for Future Networks, 2012

[4] [ITU-T Y.fsul] Draft Recommendation ITU-T Y.fsul (2014), Framework and Scenarios for Self-directed Ubiquitous Learning, 2014.

[5] S. De, P. Barnaghi, M. Bauer, S. Meissner, "Service Modelling for the Internet of Things," Federated Conference on Computer Science and Information Systems (FedCSIS), 2011

[6] Y. J. Kim, Y. K. Jeon, I. Y. Chong, "Device Objectification and Orchestration Mechanism for IoT Intelligent Service," Journal of KICS: Convergence Technologies, Vol. 38C, No. 1, pp. 19-32, Jan. 2013

[7] S. H. Lee, I. Y. Chong, "User-centric intelligence provisioning in webof-objects based IoT service,” ICT Convergence (ICTC), 2013

Young Jun Kim received Master degree from Hankuk University of Foreign Studies (HUFS) in 2003, and now he is in doctorate program of computer and information communications engineering as $\mathrm{PhD}$. candidate at HUFS. His research interests are communications service platform and WoO (Web of Objects) services. He has 3 patents and 15 contributions to ITU-T. He has published 9 papers for journals and conferences.

Sok Pal Cho received a Ph.D.(1992) from the Kyung Hee University,

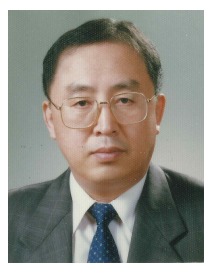
Seoul Korea. He was a computer system engineer at Control Data Corp. since 1976. He was senior researcher at R\&D Center, Sam Sung Co., from April 1984 to February, 1994. He was an assistant professor in the Division of Information and Data Communication at Nam Seoul University, Korea in 1994. He joined the department of Computer Communication Engineering, the Sungkyul University, Korea since 1995. Currently he is a professor of Telecommunication engineering and a vice president, Sungkyul University, Korea. He contributed 30 contributions regarding Y.iras (IT Risk Analysis Service) and Y.ctmp ( Convergence Terminal in multiple network and application service provider environment) to ITU-T. He has published 56 papers and presented 13 documents on SIEC International Conference. He is a senior member of IEEE since 1995, a life member and vice president (1997 2007) of KIISC(Korea Institute of Information Security \& Cryptology) since 1997, His interests are Network Security, Network Protocol, ATM and MPLS switching, E-learning, Next Generation Network, Future Network, and IT Risk Management.

Ilyoung Chong (M'92-SM'08-FM) received his Ph.D. (1993) from the

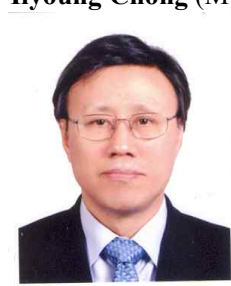
University of Massachusetts, USA. Between 1980 and 1996 he is a principal research member at ETRI (Electronics and Telecommunications Research Institute), Korea. His research interests are communications service platform, service-oriented networking and WoT/IoT (Web of Things/Internet of Things) services and project leader of Korean Consortium for ITEA2 EU project, Web of Objects (WoO). He has 27 patents (including national and international) 
and develops 6 international standards for the areas of VPN, home network and service overlay networking, and 196 contributions to ITU-

T, IEEE and ITEF. He has published 46 paper publications in refereed international journals and conferences, and 79 for national journals and conferences.

He was Chief of Editor, LNCS of Springer for 4 years since 2002, Organizing Chair, and General Chair and Steering Board of the ICOIN
(International Conference on Information Networking) (2002-2005)

He has been nominated Operating Committee Chair of IPTV Forum

Korea since 2005, and the Chair of OSIA (Open Standards of Internet Association) in 2008. He was General Chair of KRNet (Korea Internet) Conference (2009-2010), and Steering Committee Board of ICUFN (International Conference and Ubiquitous and Future Network) (20072013). 